\title{
Efficacy of rivastigmine on activities of daily living in Sri Lankan patients with Alzheimer disease and on improving caregiver burden: a prospective study
}

\author{
HA de Silva', A Pathmeswaran ${ }^{2}$ and SB Gunatilake ${ }^{3}$ \\ (Index Words: AChE inhibitor, ADL Index, caregiver demographics, Caregiver Stress Scale)
}

\begin{abstract}
Objective This open label, parallel group, prospective cohort study investigated the efficacy of rivastigmine treatment on activities of daily living (ADL) in patients with mild to moderate Alzheimer's disease (AD) and the possible benefits of this therapy on caregiver stress levels.

Methods Thirty eight consecutive patients with mild to moderate $A D$ were recruited; 22 received rivastigmine 3-6 mg twice daily (treatment group) for 20 weeks. Sixteen patients who did not receive rivastigmine served as the control group. The 17-item ADL Index was used to assess ADL and to determine the presence of functional deterioration. Caregivers were evaluated with the Caregiver Stress Scale (CSS). Each patient was required to have a committed caregiver and all caregivers were interviewed and administered the ADL Index and the Caregiver Stress Scale (CSS) at the start of treatment (week 0) and at the end of 20 weeks of treatment (week 20).

Results Patients in the control group showed a significant decline in ADL Index score at 20 weeks compared to rivastigmine-treated patients (difference in mean $A D L$ Index score $=8.5 ; p<0.001$ ). At week 20, mean change from baseline scores for CSS total and individual domain scores were better for caregivers in the treatment group than those in the control group (CSS total mean difference =19.2).
\end{abstract}

Conclusion We conclude that treatment of $A D$ patients with rivastigmine for 20 weeks produces a significant improvement in patient ADL functioning, and lower levels of caregiver stress.

\section{Introduction}

Establishing a diagnosis of AD requires not only decline in cognitive function, but also that this decline is associated with functional loss resulting in impairment of activities of daily living (ADL) [1,2]. A range of deficits in cognition, particularly in executive functioning, adversely affects the ability of AD patients to perform ADL [3]. The loss of ADL typically occurs in a hierarchical fashion, beginning with instrumental activities of daily living (e.g. housekeeping, managing finances) followed by the loss of basic activities of daily living (e.g. dressing, bathing) [3].

As a result, caregivers experience the burden of progressively increasing daily care required by the patient. In the later stages of $\mathrm{AD}$, the caregiver may have to stop working to care full-time for the patient, leading to financial hardship and a variety of emotional stresses, such as depression and anxiety [4]. Treatments for AD should improve not only higher order cognitive functions, but also basic functional abilities, which affect patient independence. Treatment with donepezil, an acetylcholinesterase (AChE) inhibitor, has been shown to improve functional abilities in $\mathrm{AD}$ patients and reduce caregiver burden and stress [5].

We recently reported a high dementia prevalence of $4 \%$ from a regional community survey of people over 65 years in Ragama, Sri Lanka, and more than $70 \%$ of these were of the Alzheimer type [6]. In the absence of formal services such as nursing homes for AD patients in Sri Lanka, there is a heavy reliance on informal and, in particular, family care for these patients. Furthermore, there is no research evidence of the practical, emotional, and economic impact of caring for a family member with dementia in Sri Lanka.

At present, there is no research evidence of benefit in treating our patients with rivastigmine. The present study investigated the efficacy of rivastigmine on functional ability in patients with mild to moderate $\mathrm{AD}$, and the related benefits to their caregivers regarding levels of caregiving stress.

\section{Methods}

\section{Study design}

This study was a 20-week, non-randomised, open label, parallel group, prospective cohort study conducted from February to November 2003. Thirty eight consecutive patients with mild to moderate $\mathrm{AD}$ presenting to a memory clinic were recruited. The diagnosis was discussed with the patients and their families, and the drug, rivastigmine, was offered to all. Twenty two patients who decided to start treatment (treatment group) received rivastigmine $1.5 \mathrm{mg}$ twice a day for 3 weeks, administered immediately after breakfast and dinner. This was followed by dose increments of $1.5 \mathrm{mg}$ twice a day, at intervals of 3 weeks, up to a maximum of $6 \mathrm{mg}$ twice a day. The dose was reduced to $3 \mathrm{mg}$ twice a day (lowest dose allowed) based on clinical judgement to improve tolerability when necessary. Sixteen patients declined treatment because they could not afford the drug. These patients served as the control group. All patients underwent regular follow up and standard clinical assessment.

${ }^{1}$ Departments of Pharmacology, ${ }^{2}$ Community Medicine and ${ }^{3}$ Medicine, Faculty of Medicine, University of Kelaniya. Correspondence: HA de S, Tel: + 9410112956 188, e-mail: <asita@mfac.kln.ac.lk> (Competing interests: none declared). Received 6 February 2005 and accepted 26 April 2005. 
Written informed consent was obtained to collect, analyse and publish findings of the study from patients, or if the patient was not capable of providing informed consent, from a member of his/her family.

\section{Patients}

Patients met standard diagnostic criteria for AD [2]. All had a Sinhala Mini Mental State Examination (S-MMSE) [7] score of 12 to 21 indicating mild to moderate disease. In addition to cognitive assessment with the S-MMSE and Cambridge Examination for Mental Disorders of the Elderly (CAMDEX) [8], all patients underwent a standardised diagnostic protocol that included:

1. Caregiver interview using the ADL Index [9] to assess evidence for functional limitation.

2. Full physical and neurological examination.

3. Biochemical analysis of blood and axial CT scans of the brain to exclude other causes of dementia.

All patients were living at home with at least one member of the family, most often the spouse. Patients with severe dementia, confined to a wheelchair, requiring 24hour skilled nursing care, with severe physical disability, with any contraindication to the drug were excluded. Most concomitant medications were allowed except those with notable cholinomimetic or anticholinergic effects, neuroleptics, and antidepressants. Any patient who showed evidence of deterioration, especially behaviour disturbances, during the study was offered appropriate treatment.

\section{Caregivers}

Each patient was required to have a committed caregiver who accompanied the patient to each clinic visit. The caregiver maintained contact with the patient for a minimum of 6 hours a day and five times a week. All caregivers except one were family members (usually a spouse or child).

\section{Outcome measures}

All caregivers were interviewed and administered the ADL Index and the Caregiver Stress Scale (CSS) at the start of treatment (week 0) and at the end of 20 weeks of treatment.

The 17-item ADL Index [9] was used to assess the regular activities and functions expected of our elderly population, and to determine the presence of functional deterioration, and each item is scored as follows: $1=$ activity performed without any assistance; 2 = activity performed with some physical or verbal assistance; $3=$ complete inability to perform activity even with assistance or refusal to perform activity. The minimum possible score of 17 indicates no apparent handicap, while the maximum possible score of 51 indicates total disability and total dependence on a caregiver. All patients recruited to this study had an ADL Index score $\leq 34$ indicating the presence of some measurable functional autonomy at baseline.
The CSS assesses caregiver hardships, stress and the level of fatigue $[5,10]$. This scale assesses a wide range of stresses directly attributable to caregiving in AD including relational and social deprivation (personal development), physical and emotional health, and jobcaregiving conflict. Each item assessing various aspects of caregiver stress has five possible responses ranging from zero (no stress) to four (constant stress).

\section{Results}

There were no significant differences between the two patient groups with regard to demographic characteristics. Mean age of the patients in this study was 71.2 and 69.4 in the treatment and control groups. There were no significant differences between the two groups with regard to disease severity and functional impairment. The baseline mean ( \pm standard deviation) SMMSE scores were 17.2 (SD \pm 0.4 ) for the treatment group and $19.3(\mathrm{SD} \pm 0.8)$ for the control group, and the baseline ADL Index scores were 27.3 (SD \pm 3.5$)$ for the treatment group and $26(\mathrm{SD} \pm 3.8)$ for the control group. Caregiver demographics, including age, sex, and relationship to patient were similar in the two groups (Table 1). All patients completed the study, and two patients in the control group required rescue medication with risperidone $1 \mathrm{mg}$ twice a day during the study period. Eleven patients in the treatment group were given the maximum dose of rivastigmine $(6 \mathrm{mg}$ twice a day). Due to significant gastrointestinal adverse effects, eight patients in the treatment group required dose reduction to $4.5 \mathrm{mg}$ twice a day, and three patients to $3 \mathrm{mg}$ twice a day.

Patients in the control group showed a significant decline in the ADL Index score at 20 weeks compared to rivastigmine-treated patients (difference in mean ADL Index score $=8.5 ; \mathrm{p}<0.001$ ). In the treatment group, nine patients showed an improvement, 12 remained at baseline,

\section{Table1. Caregiver demographics and characteristics by} treatment group

\begin{tabular}{lcc}
\hline Characteristic & $\begin{array}{c}\text { Treatment group } \\
(n=22)\end{array}$ & $\begin{array}{c}\text { Control group } \\
(n=16)\end{array}$ \\
\hline Age (years) & & \\
$\quad$ Mean \pm SD & $64.3 \pm 14.2$ & $66.8 \pm 12.9$ \\
$\quad$ Range & $32-77$ & $29-69$ \\
Female, $n(\%)$ & $14(63 \%)$ & $8(50 \%)$ \\
Relationship to patient, $\mathrm{n}(\%)$ & & \\
$\quad$ Spouse & $16(73)$ & $10(63)$ \\
$\quad$ Child / grandchild & $5(23)$ & $6(37)$ \\
$\quad$ Other & $1(4)$ & - \\
Months as caregiver & & \\
$\quad$ Mean \pm SD & $10.1(3.2)$ & $8.4(2.4)$ \\
$\quad$ Range & $4-18$ & $3-24$ \\
Level of education & & \\
$\quad<6$ years & $11(50)$ & $7(44)$ \\
$\geq 6$ years & $11(50)$ & $9(56)$ \\
Employed, $\mathrm{n}(\%)$ & $13(59)$ & $7(44)$ \\
\hline
\end{tabular}


and one showed deterioration in the ADL Index scores at 20 weeks compared to baseline score. In the control group none showed an improvement and only two remained at baseline, while 12 patients showed deterioration in the ADL Index scores.

Caregiver stress levels were similar in the two groups at baseline (Table 2). The mean change from baseline for individual items as well as total CSS scores improved or remained close to baseline at 20 weeks for the rivastigminetreated group, whereas they declined in the control group (Table 3). All individual items and the total stress score demonstrated significant benefits for caregivers of the rivastigmine group over those of the control group (Table 3 ).

Table 2. Caregiver Stress Scale (CSS): Mean (SD) total and individual domain scores at baseline

\begin{tabular}{lcc}
\hline CSS item & $\begin{array}{c}\text { Treatment group } \\
(n=22)\end{array}$ & $\begin{array}{c}\text { Control group } \\
(n=16)\end{array}$ \\
\hline $\begin{array}{l}\text { Time dependency (overload) } \\
\text { Personal development } \\
\text { (role captivity) }\end{array}$ & $11.9(2.9)$ & $12.1(3.1)$ \\
$\begin{array}{l}\text { Physical health } \\
\text { Social relationship deprivation } \\
\text { (relational deprivation) }\end{array}$ & $6.5(2.7)$ & $7.7(2.8)$ \\
$\begin{array}{l}\text { Emotional health } \\
\text { Total caregiver status }\end{array}$ & $6.4(3.3)$ & $6.1(2.3)$ \\
\hline
\end{tabular}

(These differences were not significant)

Table 3. Caregiver Stress Scale (CSS): Mean (SD) total and individual domain scores, and differences between the two groups at 20 weeks

\begin{tabular}{lcccc}
\hline CSS item & $\begin{array}{c}\text { Treatment } \\
\text { group } \\
(n=22)\end{array}$ & $\begin{array}{c}\text { Control } \\
\text { group } \\
(n=16)\end{array}$ & Difference $*_{p}$ value \\
\hline Overload & $8.4(2.7)$ & $14.7(1.6)$ & 6.3 & $<0.001$ \\
$\begin{array}{l}\text { Role captivity } \\
\text { Physical health }\end{array}$ & $6.0(2.2)$ & $9.6(2)$ & 3.6 & $<0.001$ \\
$\begin{array}{l}\text { Relational } \\
\text { deprivation }\end{array}$ & $6.0(2.5)$ & $7.5(1.8)$ & 3.0 & $<0.001$ \\
$\begin{array}{l}\text { Emotional health } \\
\text { Total caregiver }\end{array}$ & $4.3(2.2)$ & $8.4(1.5)$ & 4.9 & 0.02 \\
status & $29.1(8.9)$ & $48.3(4.9)$ & 19.2 & $<0.0001$ \\
& & & & \\
\hline
\end{tabular}

*p values based on independent samples t test

\section{Discussion}

Although the effect of AChE inhibitors on functional ability in patients with AD has been reported previously from other countries [5,11-13], the present study is the first to demonstrate the efficacy of rivastigmine in improving functional ability in patients with mild to moderate AD in Sri Lanka. Our study data shows that in Sri Lankan patients with mild to moderate $\mathrm{AD}$, rivastigmine preserves functional ability and slows the rate of decline for at least 20 weeks. There is evidence of this effect from the functional assessment measure used in this study, the ADL Index, which demonstrated an overall improvement on components required for the completion of each ADL, including initiation, planning, organisation, and effective performance in the treatment group.

Caring for patients with dementia is associated with substantial psychological strain, and progressive functional disability in these patients drives caregiver stress $[4,14$, 15]. Most caregivers in developing countries are older women caring for their husbands or younger women caring for a parent or grandparent [14]. Treatment of AD patients with rivastigmine reduces the burden and stress levels in caregivers. Caregivers of rivastigmine-treated patients reported significantly lower levels of overload, role captivity, job-caregiving conflict, social relationship deprivation, physical and emotional strain, and loss of self, than those of the control group. It is noteworthy that the smallest reduction in caregiver stress between the two groups was in the domain of social relationship deprivation, probably due to the cultural background of caregivers in our country who accept the role of caring for family elders as a social duty.

There are some limitations of the present study. Firstly, this was an open-label study where patients were not randomised to the two groups. This is because rivastigmine, the only specific treatment available for $\mathrm{AD}$ in Sri Lanka, is expensive and currently not available from the national health service. Therefore, although all patients recruited to the study were recommended treatment with rivastigmine, only those who could afford the cost of the drug were able to take it. Secondly, all key measures of ADL functioning used in the study were based on caregiver ratings and observations, and the validity of these subjective ratings and their concordance with actual observations of functional abilities has not been established. However, all patients recruited, completed the study, and the final caregiver interviews and ratings at 20 weeks were done by the principal investigator (HAdeS) in an out-patient setting by appointment, thus reducing opportunities for carers and patients of the two groups to meet each other.

Improving patient ADL functioning is one of the most important aspects of dementia treatment. Due to progressive loss of ADL in AD, improvement, maintenance, or even slowed decline of functional abilities should be considered a positive treatment outcome. Our results show that treatment of AD patients with rivastigmine for 20 weeks produces a significant improvement in ADL functioning and reduced caregiver burden. Randomised, controlled trials with AChE inhibitors in other parts of the world have shown similar results, and such trials of long term treatment, investigating all aspects of dementia care, are needed from Sri Lanka. For the present, we conclude that with rivastigmine therapy, clinicians can offer both AD patients and their caregivers in Sri Lanka an enhanced quality of life. 


\section{References}

1. American Psychiatric Association. Diagnostic and Statistical Manual of Mental Disorders, 4th ed. Washington DC: American Psychiatric Association, 1994

2. McKhann G, Drachman D, Folstein M, Katzman R, Price $\mathrm{D}$, et. al. Clinical diagnosis of Alzheimer's disease: report of the NINCDS-ADRDA work group. Neurology 1984; 34: $939-44$

3. Feldman H, Sauter A, Donald A, Gelinas I Gauthier S, et al. The Disability Assessment of Dementia Scale. A 12month study of functional ability in mild to moderate severity Alzheimer's disease. Alzheimer's Disease and Associated Disorders 2001; 15: 89-95

4. Schulz R, O'Brien AT, Bookwala J, Fleissner K. Psychiatric and physical morbidity effects of dementia caregiving. Prevalence, correlates, and causes. Gerontologist 1995; 35: 771-91

5. Feldman H, Gauthier S, Hecker J, Vellas B, Emir B, et al. Efficacy of donepezil on maintenance of activities of daily living in patients with moderate to severe Alzheimer's disease and effect on caregiver burden. Journal of the American Geriatric Society 2003; 51: 737-44

6. de Silva HA, Gunatilake SB, Smith AD. Prevalence of dementia in a semi-urban community in Sri Lanka: report from a regional survey. International Journal of Geriatric Psychiatry 2003: 18: 711-5

7. de Silva HA, Gunatilake SB. Mini Mental State Examination in Sinhalese: a sensitive test to screen for dementia in Sri Lanka. International Journal of Geriatric Psychiatry 2002; 17: 134-9
8. Roth M, Tym E, Mountjoy CQ, Huper t F A., Hendrie H, et al. CAMDEX: A standardised instrument for the diagnosis of mental disorder in the elderly with special reference to the early detection of dementia. British Journal of Psychiatry 1986; 149: 698-709

9. Sheikh K, Smith DS, Meade TW, Goldenberg E, Brennan PJ, et. al. Repeatability and validity of a modified Activities of Daily Living (ADL) Index in studies of chronic disability. International Rehabilitation Medicine 1979; 1: 51-8

10. Pearlin LI, Mullan JT, Semple SJ, Skaff M M, Caregiving and the stress process: an overview of their concepts and measures. Gerontologist 1990; 30: 583-94

11. Rösler M, Anand R, Cicin-Stain A, Gauthier S, Agid Y, et al. Efficacy and safety of rivastigmine in patients with Alzheimer's disease: international randomized controlled trial. British Medical Journal 1999; 318: 633-40

12. Tariot PN, Solomon PR, Morris JC, et al. A 5-month, randomized, placebo-controlled trial of galantamine in AD. The Galantamine USA-10 Study Group. Neurology 2000; 54: $2269-76$

13. Mohs RC, Doody RS, Morris JC, Ieni J R, Rogres SL, et al. A 1-year placebo-controlled preservation of function survival study of donepezil in AD patients. Neurology 2001; 57: 481-8

14. The $10 / 66$ Dementia Research Group. Care arrangements for people with dementia in developing countries. International Journal of Geriatric Psychiatry 2004; 19: 170-7

15. Schneider J, Murray J, Banerjee S, Mann A. EUROCARE: a cross national study of co-resident spouse carers for people with Alzheimer's disease: I-Factors associated with carer burden. International Journal of Geriatric Psychiatry 1999; 14: 651-61 